\title{
DUVEX: an x-ray counting system based on YAG:Ce scintillator
}

\author{
J-M. André, K. Le Guen, P. Jonnard \\ Laboratoire de Chimie Physique - Matière et Rayonnement, UPMC, CNRS UMR 7614, \\ 11 rue Pierre et Marie Curie, F-75231 Paris CEDEX 05, France \\ Y.Ménesguen \\ CEA, LIST, Laboratoire National Henri Becquerel, F-91191 Gif-sur-Yvette, France
}

\begin{abstract}
A detector system, called DUVEX, has been developed for the soft-x-ray and extreme ultraviolet domain. It consists in a YAG :Ce scintillator coupled to a photomultiplier module working under vacuum in counting mode. The design and the performances of this detector in terms of yield, absolute efficiency, response and noise are reported. Spectra in the soft $\mathrm{X}$-ray range of different elements ( $\mathrm{W}, \mathrm{Ag} \mathrm{Al}, \mathrm{Mg}, \mathrm{Cu}, \mathrm{N}, \mathrm{C}, \mathrm{B}$ ) obtained in WDS mode using this detector are presented. DUVEX appears as a competitive detection tool in terms of cost and easiness of implementation.
\end{abstract}

Corresponding author : Dr.J-M. André ; jean-michel.andre1@upmc.fr

Keywords : soft-X-ray, Extreme-UV, counting system, scintillator 


\section{1/ Introduction}

This work was initially motivated by the development of a counting system dedicated to soft-x-ray and E-UV (Extreme UV) radiation, in particular at the $30.4 \mathrm{~nm}$ wavelength $(40.75 \mathrm{eV})$ corresponding to the He II emission line and compatible with radiation sources emitting parasitic visible light. Such sources are discharge lamps or modified Coolidge x-ray tube as the one setup in our laboratory reflectometer [1], both of them being a source of relatively intense visible light flux. Standard detectors for this spectral domain such as photodiodes or channeltrons were unsuccessfully tested for this purpose. Diodes such as AUVX100 [2] equipped with a $150 \mathrm{~nm}$ aluminum filter were not sufficiently efficient and the short wavelength radiation was lost in the visible light without possible spectral discrimination. Channel photomultipliers (channeltrons or MCPs) are sensitive to helium leakage coming from the discharge lamp and may have an unstable response. Nevertheless a photon detector developped by Quantar Technology Inc. consisting in five MCPs and a resistive anode encoder was successfully used at $30.4 \mathrm{~nm}$ for optical measurements with a flow lamp filled with helium [3]. Standard gas-flow (Geiger-Müller) detectors usually implemented in hard $x$-ray reflectometry are inefficient because their windows are too opaque to the soft-X-ray and E-UV radiation.

Indeed development of E-UV detectors in this context remains a challenge. Recently promising results were obtained with a $\mathrm{AlGaN}$-on-Si inverted Schottky photodiode providing a cut-off wavelength of $280 \mathrm{~nm}$ with three orders of magnitude intrinsic rejection of the visible radiation [4]. Nevertheless the fabrication of this detector requires access and mastering of high technology such as molecular beam epitaxy.

Since inorganic scintillators are efficient in the spectral domain of interest and compact photomultiplier tubes (PMT) are now available at reasonable costs, we decide to built and test a counting system based on the coupling of these devices. Because of its performances, especially in terms of quantum yield, and its commercial availability, YAG doped with Ce (YAG:Ce) was chosen as scintillator. The H8259-01 PMT module supplied by Hamamatsu was retained because its peak sensitivity is well tuned to the peak emission line of the YAG:Ce and because of its compactness.

The counting system was called DUVEX (Détecteur pour l'UV Extrême et les X mous). The paper describes the design of this detector and as a first step presents its performances in terms of yield, absolute efficiency, response and noise in the soft x-ray range. Spectra of different elements obtained in WDS mode using this detector are reported. Perspectives for the improvement of the system are presented.

\section{2/ Synoptic of the counting system}

The system consists in four parts:

- a YAG:Ce scintillator crystal to convert the soft x-ray or E-UV radiation into visible light;

- a chromatic filter to select the green radiation emitted by the scintillator and reject a part of the parasitic visible light;

- a PMT coupled to the scintillator, for the readout;

- a visible filter to cut a large part of the parasitic visible light.

The scheme of the system is displayed on figure 1 . 


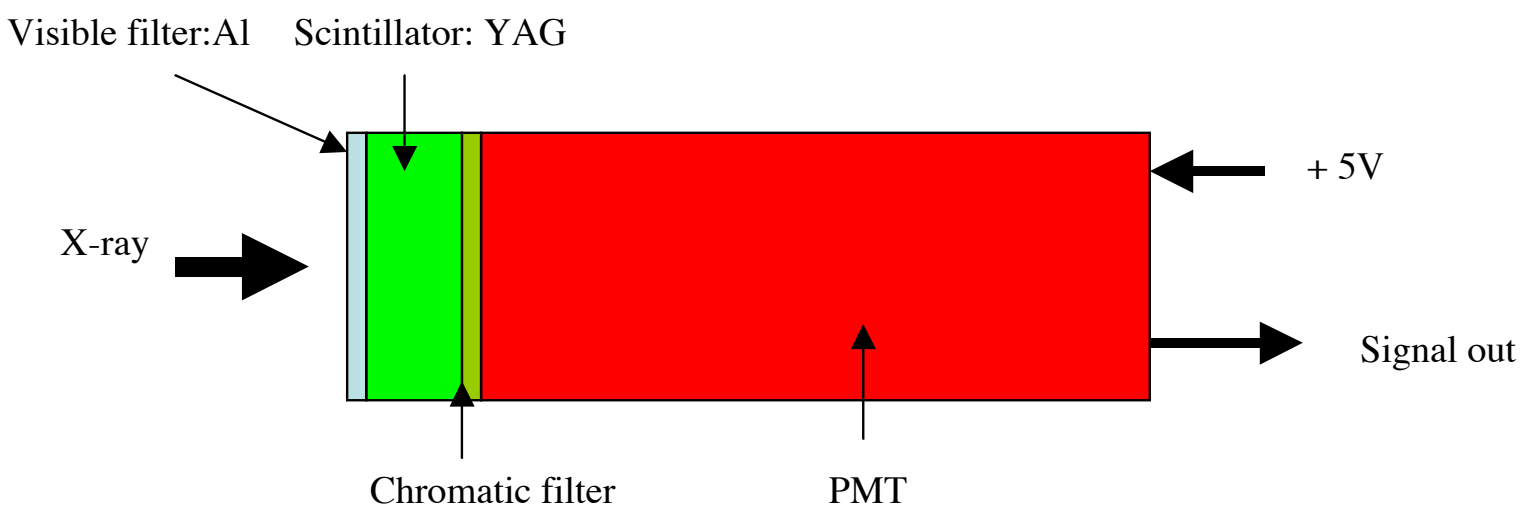

Figure 1: Counting unit based on a 150 nm-thick Al window, a YAG:Ce scintillator and a $P M T$.

\subsection{YAG:Ce scintillator}

The yttrium aluminum garnet (YAG) of chemical formula $\mathrm{Y}_{3} \mathrm{Al}_{5} \mathrm{O}_{12}$ activated by cerium is a fast scintillator with excellent mechanical and chemical resistance and is commercially available; we use a plate supplied by Crytur [5]. The decay time is around $70 \mathrm{~ns}$ that makes possible a high counting rate and the quantum yield is estimated to be between 40 and $50 \mathrm{ph} / \mathrm{keV}$ [6]. Its non-hygroscopic nature and its short decay time is advantageous compared to the standard CsI:Tl scintillator in spite of a smaller light output (factor 3). The wavelength of maximum emission (around $550 \mathrm{~nm}$ ) is well suited to the sensitivity of the photocathode of the PMT module.

Let us recall briefly that the scintillating mechanism is extremely complex but can be globally regarded as a tri-step process :

- absorption of a high-energy photon with creation of a large number of electrons-holes $(\mathrm{e}-\mathrm{h})$ pairs which relax respectively to the bottom of the conduction band and to the top of the valence band;

- relaxation of the electrons and holes toward to the luminescence centre (LC);

- luminescence of the LC.

Globally the number of visible photons $\mathrm{N}_{\mathrm{ph}}$ emitted when an amount of energy $\mathrm{E}$ is released in a scintillator upon interaction with a high energy photon is given by:

$$
\mathrm{N}_{\mathrm{ph}}=\mathrm{CE}=\frac{\mathrm{QS}}{\beta \mathrm{E}_{\text {gap }}} \mathrm{E}
$$

where $E_{g a p}$ is the value of the forbidden band gap and $\beta$ is a parameter for the average energy required to produce one thermalized electron-hole pair; $\mathrm{S}$ is the transport/transfer efficiency of the $\mathrm{e}-\mathrm{h}$ pair/energy to the LC and $\mathrm{Q}$ is the quantum efficiency of the LC, i.e. the efficiency for photon emission once the LC is excited. S depends very much on defects other than the LC, that may capture electrons or holes or both. The $\mathrm{Ce}^{3+}$ ion acts as LC. Doping with $\mathrm{Ce}^{3+}$ ions produces LCs that emit light with a sufficiently high quantum yield and possess a short radiative lifetime. This ion has one electron in the $4 \mathrm{f}$ state that is promoted to the empty $5 \mathrm{~d}$ shell upon excitation. Subsequent de-excitation will occur by an allowed $5 \mathrm{~d}-4 \mathrm{f}$ electric dipole transition with a decay time longer than $30 \mathrm{~ns}$ [7].

\subsection{Chromatic filter}

To reject most of the visible light and select mainly the green radiation centered 
around $550 \mathrm{~nm}$ emitted by the scintillator, an optional chromatic filter can be put in front of the scintillator window. We use the filter Kodak Wratten $\mathrm{n}^{\circ} 58$ which has a peak transmittance of $40 \%$ and a FWHM around $50 \mathrm{~nm}$.

\subsection{PMT module}

For the readout we choose to couple the scintillator with the H8259-01 PMT module supplied by Hamamatsu. This device consists in a photocathode, a photomultiplier, an amplifier, a discriminator and a pulse shaper giving a $2.2 \mathrm{~V}$ level output and a typical output pulse width of $10 \mathrm{~ns}$. The whole system is integrated in a single and light $(220 \mathrm{~g})$ module. We choose to operate in photon counting mode because this mode provides many advantages in comparison with the analog mode: the first one is operating stability that is a resistance to variations in supply voltage and photomultiplier tube gain; the second one is a better signalto-noise ratio (SN ratio) by a factor of the noise figure of the photomultiplier [8].

The spectral response of the photocathode is between 185 and $850 \mathrm{~nm}$ with a peak sensitivity of around $3 \mathrm{pW}^{-1} \mathrm{~s}^{-1}$ at $430 \mathrm{~nm}$, that is, not to far from the wavelength of maximum emission of the scintillator. The high voltage power supply for the photomultiplier and the discrimination level are preset to optimum level allowing photon counting by just connecting $\mathrm{a}+5 \mathrm{~V}$ supply. This simple connection together with the low weight is a great advantage because it makes easier the moving of the counting head by the arm of the goniometer. The nominal specifications of the PMT module, that are typical dark count 100 counts/s (after $30 \mathrm{~min}$ operation in darkness) and count linearity $2.510^{6}$ counts/s are promising for a correct counting dynamics (around $10^{4}$ ).

\subsection{Visible filter}

The purpose of this filter is to block as efficiently as possible the visible light emitted by the source and to transmit the maximum intensity of E-UV radiation and soft-X-rays. When using the Coolidge $\mathrm{x}$-ray tube, most of the visible light arises from the tungsten wire which is heated around $3000 \mathrm{~K}$ to produce the electrons by thermo-ionic emission; when using the discharge lamp the visible light comes from the plasma emission. The choice of the material to perform the visible cut-off is extremely reduced. Calculations show that the transmission of around $150 \mathrm{~nm}$ of aluminium is close to $80 \%$ at $30.4 \mathrm{~nm}$ and the maximum transmittance of visible light is around $510^{-8}$. Unfortunately aluminium is easily oxidized and calculation shows that alumina reduces considerably the transmission of the filter at the energy of the He II emission. Although we are aware that this way is far from being ideal, we decide to use as filter a thermally evaporated film of aluminium $150 \mathrm{~nm}$ thick directly deposited on the scintillator. Of course other material and thicker film could be used for the soft-X-ray domain but we choose to implement the same filter for both soft-X-rays and E-UV for practical convenience.

\section{3/ Calculation of the absolute efficiency}

As usual the absolute efficiency $\varepsilon$ is defined by the ratio between the number of detected $\mathrm{X}$-ray photons $\mathrm{N}^{\mathrm{X}}$ and the number of incident $\mathrm{X}$-ray photons $\mathrm{N}_{0}^{\mathrm{X}}$. Practically in the counting mode, one records the number of counts $\mathrm{N}$ at the output of the PMT module. Assuming a quasi monochromaticity of the scintillator emission, $\mathrm{N}$ is proportional to the number of visible photons $\mathrm{N}^{\mathrm{V}}$ emitted by the scintillator and impinging the window of PMT:

$$
\mathrm{N}=\mathrm{K}_{1} \mathrm{~K}_{2} \mathrm{~N}^{\mathrm{V}}
$$


The coefficient $\mathrm{K}_{1}$ is the count sensitivity CS of the PMT at the visible wavelength $(550 \mathrm{~nm})$ [9]. Since at $550 \mathrm{~nm} \mathrm{CS}$ is equal to $2.810^{5} \mathrm{pJ}^{-1}$ and the visible photon energy is $2.25 \mathrm{eV}$ that is $3.610^{-7} \mathrm{pJ}$, one finds $\mathrm{K}_{1}=0.1$. The coefficient $\mathrm{K}_{2}$ corresponds to the rate of visible photons actually produced by the detected X-rays which enter the PMT; this term is tricky to evaluate. It is roughly given by the solid angle subtended by the surface of the PTM window from the point of visible light emission; if the visible light emission is assumed to be isotropic (lambertian source) from the geometry one can estimate $\mathrm{K}_{2}$ to be close to 0.28 .

As explained in $\S 2.1, \mathrm{~N}^{\mathrm{V}}$ is proportional to the total energy $\mathrm{E}_{\text {tot }}$ deposited by the $\mathrm{x}$-ray beam that is, in case of monochromatic radiation of photon energy $\mathrm{E}$ :

$$
\mathrm{N}^{\mathrm{V}}=\mathrm{T} \text { A C E N }
$$

where $\mathrm{T}$ is the $\mathrm{x}$-ray transmittance of the visible filter at the photon energy $\mathrm{E}, \mathrm{A}$ the absorbance of the scintillator also at $\mathrm{E}$ and $\mathrm{C}$ the coefficient of transfer of the deposited $\mathrm{X}$-ray energy into the number of visible photons $\mathrm{N}^{\mathrm{V}}$. C is obtained from Eq. (1); with $\mathrm{E}_{\text {gap }}=5 \mathrm{eV}$, $\beta=2.5, Q=1$ and $S=0.5$, one has $C=0.04 \mathrm{eV}^{-1}$. One can roughly estimate the value of $\mathrm{N}_{\mathrm{ph}}$ to be around 1.6 at $40.75 \mathrm{eV}$ in agreement with the data of reference [5].

To calibrate DUVEX one measures in the same conditions with a monochromatic $\mathrm{x}$ ray, the ratio $M$ of the number of counts given by DUVEX to the number of counts $\mathrm{N}^{\mathrm{X}}$ ref given by a reference detector whose absolute efficiency $\varepsilon_{\text {ref }}$ is known (See $\S 4.1$ ); then one has:

$$
\mathrm{M}=\frac{\mathrm{N}}{\mathrm{N}_{\mathrm{ref}}^{\mathrm{X}}}=\frac{\mathrm{K}_{1} \mathrm{~K}_{2} \mathrm{~N}^{\mathrm{V}}}{\varepsilon_{\mathrm{ref}} \mathrm{N}_{0}^{\mathrm{X}}}=\frac{\mathrm{K}_{1} \mathrm{~K}_{2} \mathrm{TACEN}_{\mathrm{D}}^{\mathrm{X}}}{\varepsilon_{\mathrm{ref}} \mathrm{N}_{0}^{\mathrm{X}}}=\mathrm{K}_{1} \mathrm{~K}_{2} \mathrm{TACE} \frac{\varepsilon}{\varepsilon_{\mathrm{ref}}}
$$

and then:

$$
\varepsilon=\frac{\mathrm{M} \varepsilon_{\text {ref }}}{\mathrm{K}_{1} \mathrm{~K}_{2} \mathrm{TACE}}
$$

The yield of DUVEX is defined by the ratio $\mathrm{Y}=\mathrm{N} / \mathrm{N}^{\mathrm{X}}{ }_{0}$ that is the number of counts delivered by DUVEX related to the number of incoming photons. In terms of $\mathrm{Y}$, the absolute efficiency $\varepsilon$ is given by:

$$
\varepsilon=\frac{Y}{\mathrm{~K}_{1} \mathrm{~K}_{2} \mathrm{~T} \text { A C E }}
$$

\section{4/ Characterization of DUVEX}

\subsection{Absolute efficiency measurements in the soft $x$-ray range}

To measure the absolute efficiency in the soft-x-ray domain, we use the SOLEX facility [10]. This instrument was designed to perform absolute efficiency measurements of $\mathrm{x}$-ray detectors by recording the counting rate via two channels: one is the detector under characterization and the other is a calibrated detector. The latter (SIMEON) is a Si-Li detector supplied by Gresham. In the procedure of measurement, different corrections are applied: dead time of SIMEON, transmittance of the windows and filters ; the relative accuracy on the absolute efficiency is estimated to be around $\pm 2 \%$. 
The measurements are carried out with five emission lines: $\mathrm{Cu}-\mathrm{L} \alpha(930 \mathrm{eV} /$ $1.336 \mathrm{~nm}), \mathrm{Mg}-\mathrm{K} \alpha(1253.6 \mathrm{eV} / 0.989 \mathrm{~nm}), \mathrm{Si}-\mathrm{K} \alpha(1740 \mathrm{eV} / 0.712 \mathrm{~nm}), \mathrm{W}-\mathrm{M} \alpha(1775 \mathrm{eV} /$ $0.698 \mathrm{~nm}), \mathrm{Ag}-\mathrm{L} \alpha(2984 \mathrm{eV} / 0.416 \mathrm{~nm})$; the radiation emitted by an X-ray tube equipped with a target including the emitting chemical element is monochromatized by means of a bent $10 \overline{10}$ beryl crystal. Figure 2 shows the value of $M \cdot \varepsilon_{\text {ref }}=N / N^{X}{ }_{0}$ and $\varepsilon$ absolute efficiency of DUVEX deduced from the measurements done with SOLEX and using the formula (5) given by the model described in $\S 3$. We have calculated the transmittance $\mathrm{T}$ by means of the coefficient of absorption available in [11]; all the x-ray photons being absorbed in the scintillator we have taken $A=1$. Note that the measurements have been done without chromatic filter.

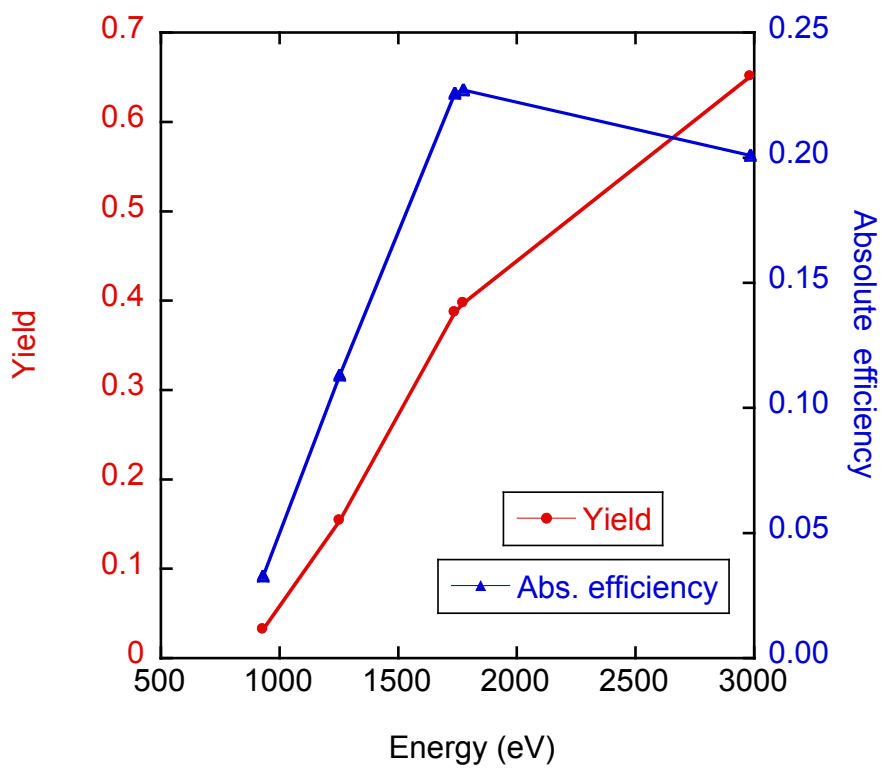

Figure 2: Absolute efficiency and yield of DUVEX at different photon energies.

\subsection{Tests at various emission lines in the soft-x-ray range}

We test the performances of the system DUVEX by means of the MONOX facility [1]. The radiation emitted by the soft-x-ray source was monochromatized with the help of crystals or multilayer Bragg mirrors setup in the monochromator unit of MONOX. At a specific emission line, we recorded the efficiency of the detector in the photon counting mode versus the current of the electron gun of the tube. Figure 3 shows the response of the detector versus the current of the electron gun of the x-ray tube at different photon energies corresponding to the emission lines of different elements: Al-K (1486 eV/ $0.834 \mathrm{~nm}), \mathrm{Mg}-\mathrm{K}$ (1253.6 eV/ $0.989 \mathrm{~nm}), \mathrm{Cu}-\mathrm{L}$ (929.7 eV/ $1.336 \mathrm{~nm}), \mathrm{N}-\mathrm{K}(392.4 \mathrm{eV} / 3.160 \mathrm{~nm}), \mathrm{C}-\mathrm{K}(277 \mathrm{eV} /$ $4.476 \mathrm{~nm})$. 


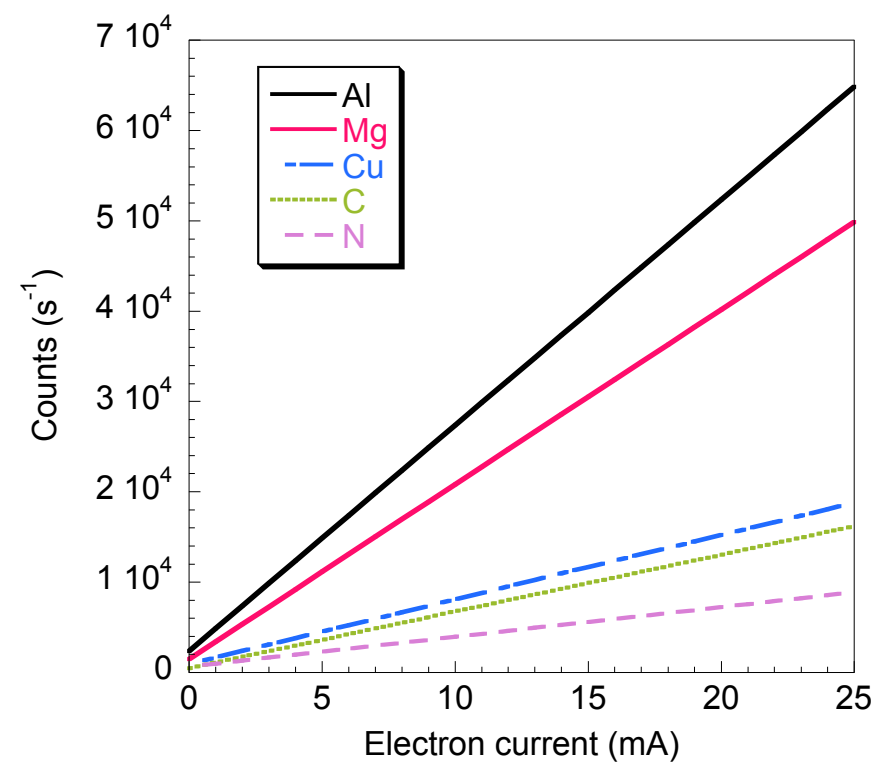

Figure 3: Output of DUVEX at different monochromatic radiations in counts/s versus the current of the electron gun of the $x$-ray tube.

We note a linear response over 4 decades and that the slope of the linear curves globally increases with the photon energy in agreement with the results of $\S 4.1$. However, a rather important background between $700 \mathrm{cps} / \mathrm{s}$ and $2300 \mathrm{cps} / \mathrm{s}$ according to the conditions of measurements is observed. We checked that a large part of this background can be attributed to intrinsic noise of the counting system while the other part is due to the visible light emitted by the source that was not blocked by the filter and to electronic noise depending on the electromagnetic environment. The intrinsic noise measured in our conditions (at least $70 \%$ of the total noise background) appears considerably larger than the typically dark count of our H8259-01 PMT module specified in the data sheet of the supplier (around 100 counts/s). This difference could be explained by a heating effect; indeed since the PTM unit operates in vacuum so that the heat dissipation is difficult, it is likely that the temperature of the module is higher than the ambient temperature at which the specified dark count was measured by the supplier.

We have recorded the emission spectra of different elements with a Bragg analyzer (multilayer mirror or crystal) in the $\theta-2 \theta$ mode of the goniometer. Figures 4 and 5 present the $\mathrm{Al}-\mathrm{K} \alpha$ and $\mathrm{N}-\mathrm{K}$ spectra respectively, after subtraction of the background. Let us mention that the detection of the N-K line with a gas-flow detector is rather difficult because of the window absorption and is really made easier with DUVEX. 


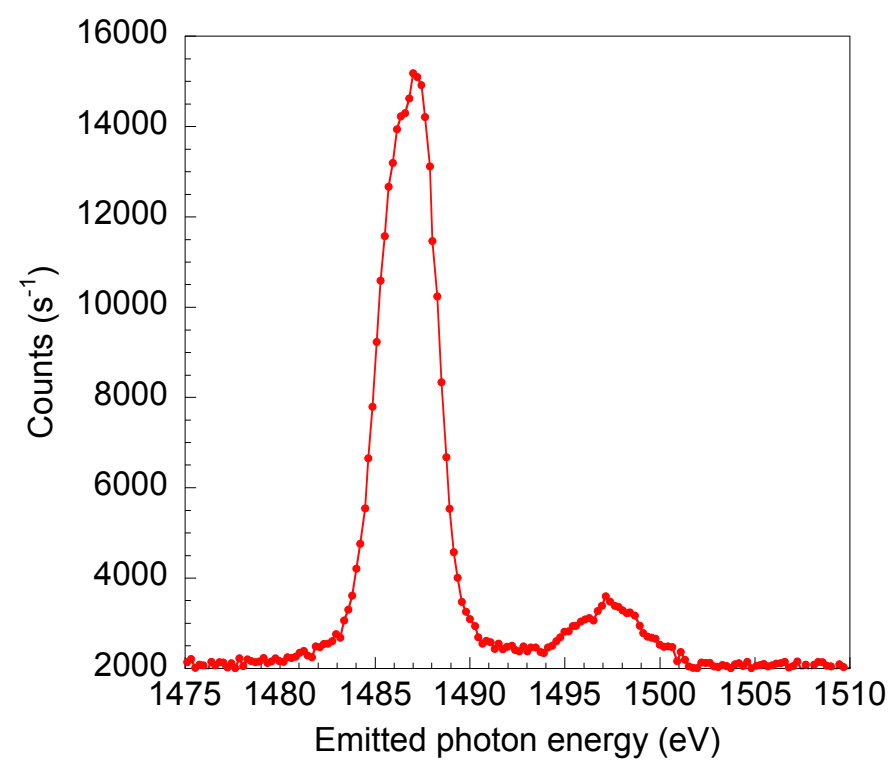

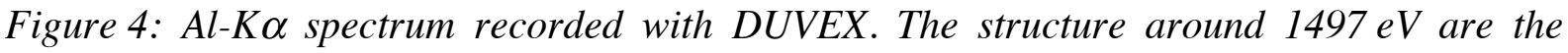
Al $K \alpha_{3,4}$ satellites.

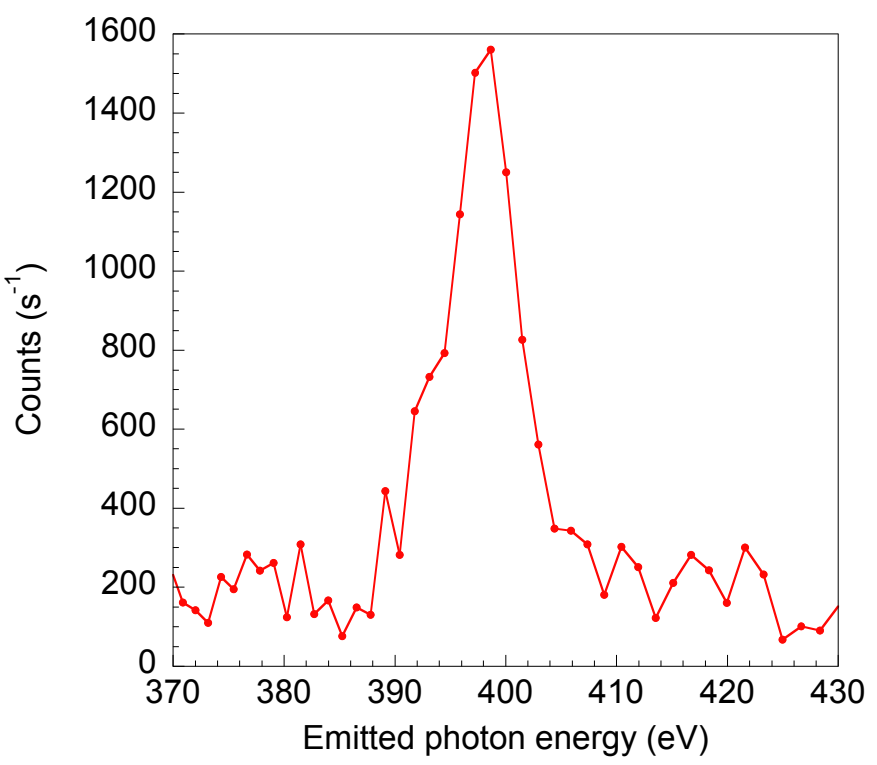

Figure 5: N-K spectrum recorded with DUVEX.

\section{5/ Conclusion and perspective}

The system DUVEX appears as an efficient and convenient counting system in the soft $\mathrm{x}$-ray domain. Its cost is considerably smaller than a counting system based on a gas-flow detector and it does not required gas with a system a pressure control for its running. The system of filters blocks efficiently the parasitic visible light. Now the system has to be evaluated in the E-UV domain by means of the He discharge lamp or synchrotron radiation. This work is underway.

In its present state, the main drawback of DUVEX is the relatively large background (dark counting) coming from an important thermal noise of the PMT that reduces the detection limit (DL). To improve the DL and the signal-to-noise ratio, one can plan to cool the PMT since the thermo-ionic emission of electrons from the photocathode and the dynodes is the major cause of dark noise. A thermoelectric cooler is under study in collaboration with the 
PMT supplier, the main problem being to prevent the moisture condensation at the input window. As another future improvement we shall replace the present filter by a $150 \mathrm{~nm}$-thick Al film sandwiched between two $5 \mathrm{~nm}$-thick carbon films. The carbon films will be deposited prior the $\mathrm{Al}$ film is put in air and will prevent the formation of the alumina.

Acknowledgements : Part of this work has been done in collaboration with Kevin, Romain, Julie et Claire students at Lycée Fresnel, Paris and Laurine student at Ecole Nationale de Chimie, Physique Biologie de Paris. M.-C. Lépy from LNHB is thanked for her help in the measurements with SOLEX. 


\section{References}

[1] J.-M. André, A. Avila, R. Barchewitz, R. Benbalagh, R. Delaunay, D. Druart, P. Jonnard, H. Ringuenet, "MONOX : a characterisation tool for the X-UV rang", Eur. Phys. J. Appl. Phys. 31, 147-152 (2005).

[2] G. Murakami, K. Sakai, T. Homma, K. Yoshioka, I. Yoshikawa, S. Ichimaru, H. Takenaka, "Performances of $\mathrm{Y}_{2} \mathrm{O}_{3} / \mathrm{Al}$ multilayer coatings for the He II radiation at 30.4 nm”, Rev. Sci. Instrum 82, 033106 (2011).

[3] P. E. Malinowski J.-Y. Duboz, P. De Moor, K. Minoglou, J. John, S. M. Horcajo, F. Semond, E. Frayssinet, P. Verhoeve, M. Esposito, B. Giordanengo, A. BenMoussa, R. Mertens, C. Van Hoof, "Extreme ultraviolet detection usingAlGaN-on-Si inverted Schottky photodiodes", Appl. Phys. Lett. 98, 141104 (2011).

[4] IRD, 2527 West $237^{\text {th }}$ Street Unit A, Torrance, CA 90505-5243; http://www.irdinc.com/index.html.

[5] CRYTUR Ltd., Palackeho 175, 51101 Turnov, Czech Republic ; http://www.crytur.cz/pages/15/crytur-materials.

[6] L. Thinova, C. Karasinski, J. Tous, T. Trojek, "Investigation of thin YAP and YAG scintillator characteristics for alpha radiation spectrometry", J. Phys. Conf. Series 41, 573-576 (2006).

[7] C. W. E. van Eijk, "Inorganic-scintillator development", Nucl. Instrum. Meth. Phys. Res. A, 460, 1-14 (2001).

[8] Hamamatsu Photonics K. K., Photomultiplier tubes: Basics and Applications, $3^{\text {rd }}$ Edition, February 2006.

[9] Hamamatsu. H8259-01 Test Sheet.

[10] C. Bonnelle, P. Jonnard, J.-M. André, A. Avila, D. Laporte, H. Ringuenet, M.-C. Lépy, J. Plagnard, L. Ferreux, J.-C. Protas, "SOLEX : a tunable monochromatic x-ray source in the 1$20 \mathrm{keV}$ energy range for metrology”, Nucl. Instrum. Meth. Phys. Res. A 516, 594-601 (2004).

[11] http://henke.lbl.gov/optical_constants/filter2.html. 